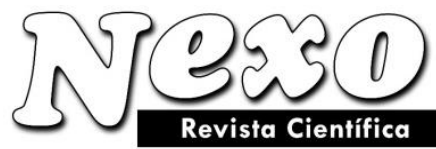

Vol. 33, No. 02, pp. 392-408/Diciembre 2020

\title{
Mineral chemistry and geothermobarometry of gabbroic rocks from the Gysel area, Alborz mountains, north Iran
}

\section{Química mineral y geotermobarometría de rocas gabroicas del área de Gysel, montañas de Alborz, Irán del norte}

\author{
Farzaneh Farahi, Saeed Taki*, Mojgan Salavati
}

Department of Geology, Faculty of Basic Sciences, Lahijan Branch, Islamic Azad University, Lahijan, Iran.

Corresponding autor email: taki_saeed2002@yahoo.com

(recibido/received: 02-June-2020; aceptado/accepted: 04-August-2020)

\begin{abstract}
The gabbroic rocks in the Gysel area of the Central Alborz Mountains in north Iran are intruded into the Eocene Volcano-sedimentary units. The main gabbroic rocks varieties include gabbro porphyry, olivine gabbro, olivine dolerite and olivine monzo-gabbro. The main minerals phases in the rocks are plagioclase and pyroxene and the chief textures are sub-hedral granular, trachytoidic, porphyritic, intergranular and poikilitic. Electron microprobe analyses on minerals in the rock samples shows that plagioclase composition ranges from labradorite to bytonite, with oscillatory and normal chemical zonings. Clinopyroxene is augite and orthopyroxene is hypersthene to ferro-hypersthene. Thermometry calculations indicate temperatures of $650^{\circ} \mathrm{C}$ to $750^{\circ} \mathrm{C}$ for plagioclase crystallization and $950^{\circ} \mathrm{C}$ to $1130^{\circ} \mathrm{C}$ for pyroxene crystallization. Clinopyroxene chemistry reveals sub-alkaline and calc-alkaline nature for the parental magma emplaced in a volcanic arc setting.
\end{abstract}

Keywords: Gabbro, mineral chemistry, thermobarometry, plagioclase, pyroxene, Alborz, Iran

\section{RESUMEN}

Las rocas gabroicas en el área de Gysel de las montañas Alborz Central en el norte de Irán se introducen en las unidades sedimentarias del volcán Eoceno. Las principales variedades de rocas gabroicas incluyen pórfido de gabro, gabro olivino, dolerita olivina y monzo-gabro olivino. Las principales fases minerales en las rocas son la plagioclasa y el piroxeno y las texturas principales son sub-hedral granular, trachytoidic, porfirita, intergranular y poikilitic. Los análisis de micro sonda electrónica sobre minerales en las muestras de roca muestran que la composición de plagioclasa varía de labradorita a bytonita, con zonificaciones químicas oscilatorias y normales. El clinopiroxeno es augita y el ortopiroxeno es hiperesteno a ferrohiperesteno. Los cálculos de termometría indican temperaturas de $650^{\circ} \mathrm{C}$ a $750^{\circ} \mathrm{C}$ para la cristalización de plagioclasa y de $950^{\circ} \mathrm{C}$ a $1130{ }^{\circ} \mathrm{C}$ para la cristalización de piroxeno. La química del clinopiroxeno revela la naturaleza subalcalina y calcálica alcalina para el magma parental emplazado en un entorno de arco volcánico. 
Palabras clave: Gabbro, química mineral, termobarometría, plagioclasa, piroxeno, Alborz, Irán

\section{INTRODUCCIÓN}

The study area is a part of the Alborz Mountains in North Iran and western part of the Central Alborz. The area is situated close to the boundary between the Central and West Alborz geological units. Gabbro is intruded as small stocks within the Eocene volcano-sedimentary units. Mineral chemistry of these gabbros are studied in order to elucidate the nature of these rocks in the context of Alborz orogeny in North Iran.

Chemical composition of mineral phases in igneous rocks, specially pyroxene can be used to decipher the physico-chemical conditions of magma crystallization (Putrika et al., 2003; Putrika, 2008), the nature of original magma (Le Bas et al., 1962; Berger et al., 2005; Dioh et al., 2009) and the tectonic setting of magmatism (Leterrier et al., 1982). Relatively young (Tertiary) magmatism in Iran along the two main orogenic belts, Zagros and Alborz are related to different events. Agard et al. (2011) consider the Tertiary volcanic-plutonic magmatism as a result of subduction of Neotethys oceanic crust beneath central Iran and subsequent collision in Oligocene. Valizadeh et al. (2008), Rahimi et al. (2010), Nemati and Asiabanha (2016) and Teimouri et al. (2018) consider a continental volcanic arc setting for Tertiary magmatism, while others propose a back-arc setting for the rocks (e.g. Asiabanha and Foden, 2012). According to studies by Verdel et al. (2011), Alborz-Azerbaijan geological unit was attached to the Urmia-Dokhtar magmatic belt up to Eocene. An inter-arc rifting separated these after Eocene. Mohajjel et al. (2014), consider this rifting as a result of post-collision extension in the continental crust.

We provide new filed, petrography and mineral chemistry data for Tertiary gabbroic rocks from the Alborz Mountains to help understanding the geodynamic evolution of this part of the Alpine-Himalayan orogenic belt.

\section{GEOLOGICAL SETTING}

The rock units presented in the study area are Paleogene to recent in age. The main part of the study area is covered by Eocene volcano-sedimentary rocks, intruded by gabbroic rocks. The Eocene deposits are consisted of volcanic rocks of basic to intermediate composition and volcanoclastic rocks of acidic and intermediate tuffs (Figure. 1). The volcanoclastic unit is red in the field outcrop. Tuffs of this unit are mainly crystalline or crystalline-lithic tuffs (Baharfiroozi et al., 2002). The composition varies from dacite to andesite with porphyroclastic textures. Microscopic studies show that a part from lithic fragments, broken fragments of feldspar, pyroxene and amphibole are present in a glass or crypto-crystalline matrix which is altered. The composition of lavas within the volcano-clastic unit is basalt, andesi-basalt, trachyandesite and andesite (Figure.1). Filed relations reveal that volcanic and volcano-clastic rocks are formed in two distinct episodes. The first was crystallization of lava in a shallow marine environment with low quantity of sedimentary rocks. The rocks of this episode are green tuffs and andesite (equivalent to some parts of the Karaj formation, Asiabanha, 2001). The second episode is depicted by intermediate to basic rocks, younger than Eocene, which are erupted in an aerial condition and lack sedimentary rocks. The rock varieties include olivine basalt, basalt, andesi-basalt and trach-andesite (Annells et al., 1975). Plutonic grabbric rocks appear mainly as small- to medium-sized stocks (from a few meters to few hundred meters) or dykes (0.5 to 2 meter thick) (Figure. 2). Gabbroic rocks are slightly to moderately altered (Figure. 2).

The stocks are chiefly gabbro in composition, while dykes are quartz-diorite. The contact between stocks and dykes with the country rocks are marked by alteration and clear contact metamorphism cannot be observed. However, the stocks and dykes clearly cut the Eocene volcano-sedimentary rocks, therefore are younger than Eocene in age. 


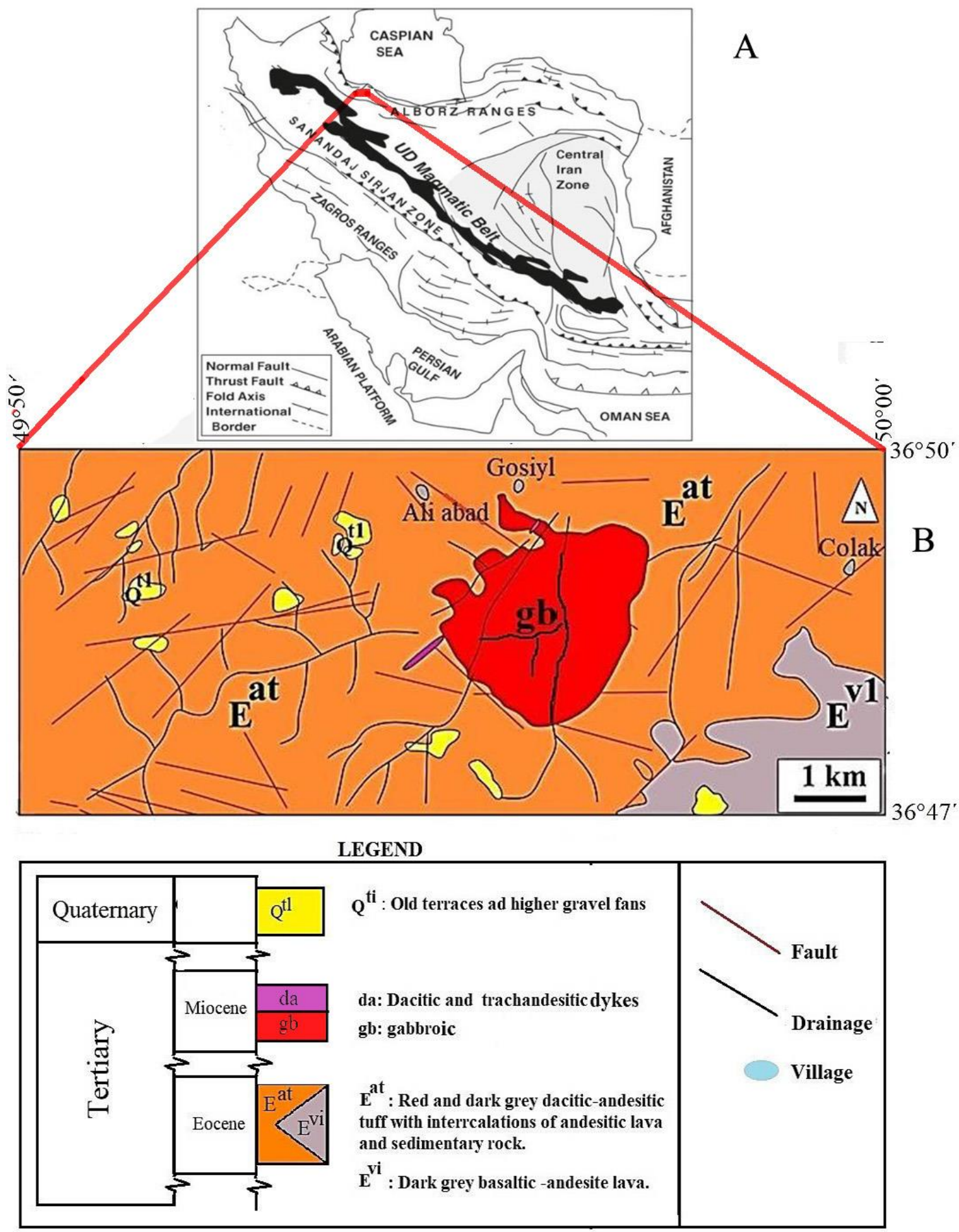

figure 1. A: Location of the study area in the geological map of Iran (Modified from Gile et al. 2006). B: Simplified geology map of the study area (after Baharfiroozi et al., 2002). 



figure 2. A: field view of gabbroic stocks about $5.5 \mathrm{~km}$ south of Gysel village. B: A view of olivine gabbro stock at $2 \mathrm{~km}$ south of Gysel village.

\section{ANAL YTICAL METHODS}

Optically well characterized and representative samples of the gabbroic rocks were chosen for electron probe micro analyses (EPMA). Mineral compositions were determined using a JEOL JXA- 8200 electron probe micro-analyser (EPMA) at the Institute of Geosciences of the University of Potsdam, Germany. The electron probe is equipped with five wavelength dispersive X-ray spectrometers (WDS). Analytical conditions were a $15 \mathrm{kV}$ accelerating potential, $2 \mu \mathrm{m}$ spot diameter and a $10 \mathrm{nA}$ beam current. The counting time for each element peak was $20 \mathrm{~s}$ and $10 \mathrm{~s}$ for peak background positions. The alkali elements $(\mathrm{Ca}, \mathrm{K}, \mathrm{Na})$ were first measured for only $10 \mathrm{~s}$ for the peaks and $5 \mathrm{~s}$ for the background in order to avoid their diffusion. All data were reduced using ZAF correction factors. Calibration against a set of synthetic oxides and natural silicates obtained from the Smithsonian Institute was repeated after every 100 measurements. The EPMA results are presented in Tables 1 to 5. 


\section{PETROGRAPHY}

The intrusive rocks are mainly gabbroic in composition. They can be divided into gabbro porphyry, olivine gabbro, olivine dolerite and olivine monzo-gabbro based on mineralogy and textures. These rocks appear as small stocks and dark green in colour. The rocks are relatively fine- to medium-grained. The main textures are sub-hedral granular, (Figure. 3a to c), porphyroid (Figure. 3b), trachytoid (Figure. 3d), intergranular and poikilitic. The main mineral phases are plagioclase and pyroxene. Plagioclase is labradorite to bytonite (optical identification and EPMA). They are slightly altered to clay minerals. Polysynthetic and Carlsbad twinnings are common. Pyroxene is mainly clinopyroxene with subordinate amounts of orthopyroxene.

Some samples contain subordinate amounts of olivine. Olivine is altered in these samples. Rare K-feldspar are fresh and fill the spaces between other minerals. They envelop plagioclase crystals occasionally. Chlorite, calcite and idddingsite are alteration products. Accessory minerals are opaque minerals, biotite and zircon.
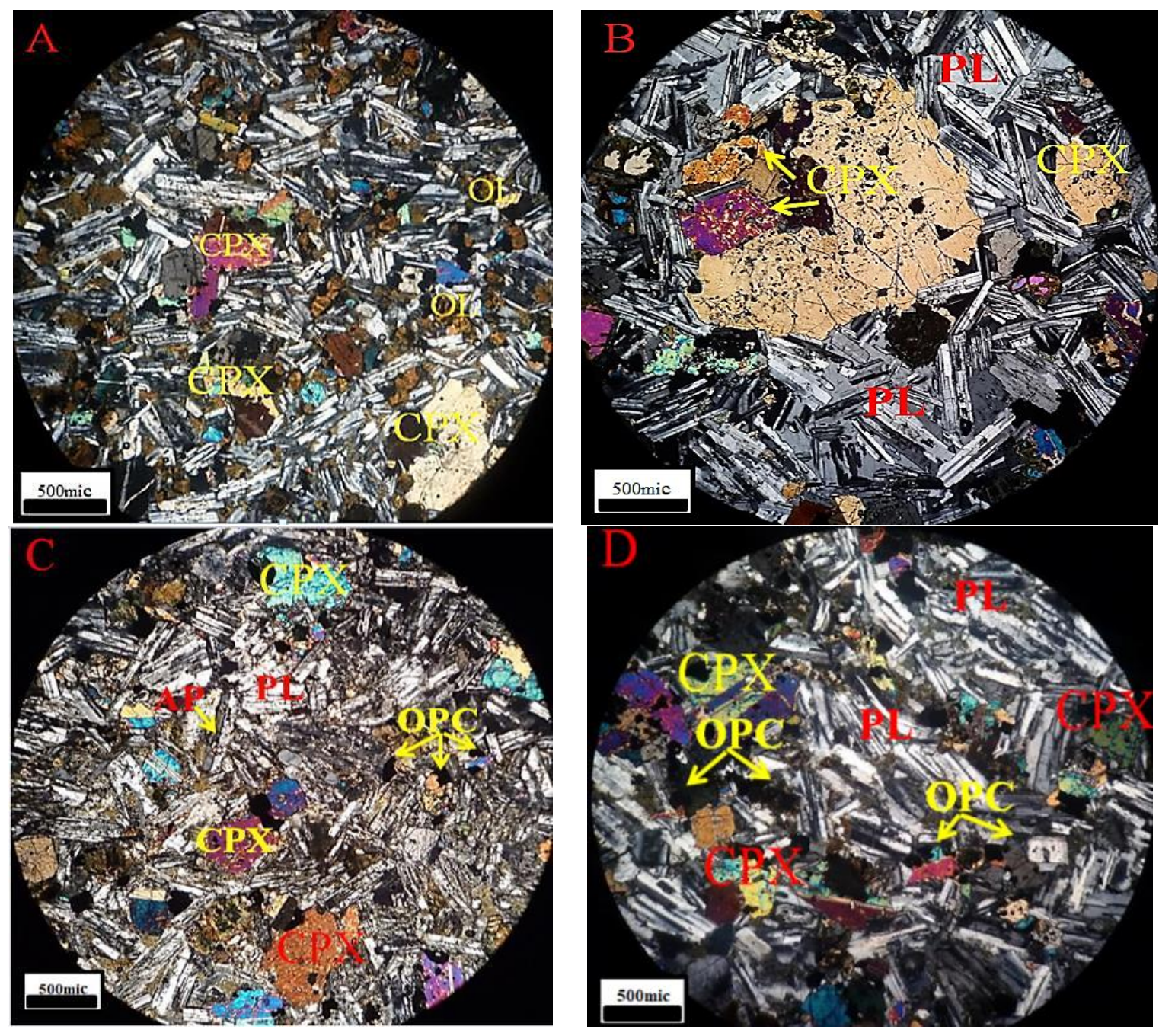

figure 3.A: Granular sub-hedral texture in relatively fine-grained olivine monzo-gabbro. B: Porphyroidic texture in olivine monzo-gabbro. C: Granular sub-hedral texture in olivine gabbro. D: Trachytoidic texture in olivine gabbro. 


\section{Mineral Chemistry}

\section{DISCUSSION}

Table 1. EPMA results of plagioclase in the gabbroic rocks.

\begin{tabular}{|c|c|c|c|c|c|c|c|c|c|c|c|}
\hline Sample & $\mathrm{Na}_{2} \mathrm{O}$ & $\mathrm{TiO}_{2}$ & MnO & $\mathrm{K}_{2} \mathrm{O}$ & $\mathbf{A l}_{2} \mathbf{O}_{3}$ & $\mathrm{Cr}_{2} \mathrm{O}_{3}$ & $\mathbf{F e O}$ & $\mathrm{SiO}_{2}$ & MgO & $\mathrm{CaO}$ & Total \\
\hline S19-PI1 & 4.08 & 0.085 & 0.004 & 0.268 & 30.412 & 0.012 & 0.357 & 52.72 & 0.029 & 12.332 & 100.3 \\
\hline S19-PI2 & 4.12 & 0.113 & 0 & 0.269 & 30.504 & 0.003 & 0.405 & 53.447 & 0.024 & 2.19 & 01.076 \\
\hline S19-PI2 & 3.98 & 0.139 & 0.026 & 0.405 & 30.093 & 0.001 & 0.468 & 53.411 & 0.063 & 2.282 & 100.866 \\
\hline S19-PI2-1 & 0.04 & 51.085 & 0.449 & 0.004 & 0.044 & 0 & 49.925 & 0.015 & 0.688 & 0.014 & 102.268 \\
\hline S19-PI2-2 & 0 & 51.216 & 0.458 & 0.011 & 0.037 & 0.017 & 49.546 & 0.025 & 0.704 & 0.024 & 102.038 \\
\hline Line 1 S19-P13 & 5.37 & 0.093 & 0.001 & 0.416 & 27.918 & 0 & 0.53 & 56.112 & 0.027 & 9.436 & 99.903 \\
\hline Line 2 S19-PI3 & 4.70 & 0.108 & 0 & 0.469 & 29 & 0.001 & 0.43 & 54.656 & 0.028 & 10.923 & 100.314 \\
\hline Line 3 S19-PI3 & 1.941 & 0.086 & 0 & 0.106 & 34.329 & 0.004 & 0.478 & 47.68 & 0.034 & 16.428 & 101.086 \\
\hline Line 4 S19-PI3 & 2.021 & 0.084 & 0.022 & 0.11 & 34.135 & 0 & 0.552 & 48.013 & 0.02 & 16.464 & 101.421 \\
\hline Line 5 S19-PI3 & 1.89 & 0.063 & 0 & 0.095 & 34.449 & 0.012 & 0.482 & 47.431 & 0.014 & 16.664 & 101.1 \\
\hline Line 6 S19-Pl3 & 2.008 & 0.054 & 0 & 0.112 & 34.045 & 0 & 0.491 & 47.826 & 0.023 & 16.334 & 100.893 \\
\hline Line 7 S19-PI3 & 1.776 & 0.04 & 0 & 0.105 & 32 & 0.017 & 0.519 & 47.083 & 0.026 & 16.808 & 100.606 \\
\hline Line 8 S19-PI3 & 2.043 & 0.049 & 0.011 & 0.117 & 33.847 & 0 & 0.477 & 47.862 & 0.036 & 16.309 & 100.751 \\
\hline Line 9 S19-Pl3 & 6.415 & 0.083 & 0 & 0.646 & 25.716 & 0.005 & 0.336 & 59 & 0.017 & 7.24 & 99.458 \\
\hline Line 10 S19-PI3 & 5.348 & 0.058 & 0 & 0.4 & 28.162 & 0.001 & 0.428 & 55.642 & 0.012 & 9.779 & 99.83 \\
\hline Line 10 S19-PI4 & 5.357 & 0.077 & 0 & 0.38 & 28.254 & 0.002 & 0.455 & 56.112 & 0.035 & 9.856 & 100.528 \\
\hline S19-PI4-1 & 4.481 & 0.08 & 0.007 & 0.406 & 28.849 & 0.001 & 0.346 & 54.299 & 0.02 & 11.219 & 08 \\
\hline S19-PI4-2 & 1.936 & 0.041 & 0 & 0.123 & 33.454 & 0.005 & 0.445 & 47.736 & 0.012 & 16.537 & 100.289 \\
\hline Line 1 S19-PI5 & 5.867 & 0.032 & 0.027 & 0.489 & 31 & 0.007 & 0.476 & 57.363 & 0.016 & 8.365 & 99.023 \\
\hline Line $2 \mathrm{~S} 1$ & 4.431 & 0.079 & 0 & & & 0 & 0.418 & 54. & 45 & 10.923 & 99.662 \\
\hline Line 3 S19-Pl5 & 2.376 & 0.089 & 0.005 & 0.169 & 249 & 0.004 & 0.512 & 48.882 & 0.013 & 15.526 & 99.825 \\
\hline Line $4 \mathrm{~S} 1$ & 1.825 & 0.101 & 0.033 & 0.106 & 33.291 & 0 & 0.476 & 47.962 & 0.022 & 16.478 & 100.294 \\
\hline Line 5 S1 & 4.541 & 0.078 & 0.013 & 0.585 & 149 & 0.028 & 0.413 & 55.201 & 0.039 & 10.853 & 99 \\
\hline Line 6 S1 & 4.546 & 0.099 & 0.003 & 0.515 & 52 & 0.008 & 0.524 & 54.02 & 0.054 & 11.228 & 99.649 \\
\hline Line 7 S19. & 4.061 & 0.055 & 0 & 0.491 & 56 & 0 & 0.39 & 53.696 & 0.028 & 11.599 & 99.276 \\
\hline Line 8 S19-PI5 & 2.237 & 0.088 & 0 & 0.168 & 32.188 & 0 & 0.486 & 48.992 & 0.027 & 15.536 & 99.722 \\
\hline Line 9 S19-PI5 & 4.907 & 0.073 & 0.008 & 0.501 & 28.01 & 0 & 0.368 & 55.253 & 0.036 & 10.455 & 99.611 \\
\hline Line 10 S19-PI5 & 5.61 & 0.064 & 0.012 & 0.422 & & 0.009 & 0.528 & 57.428 & 0.037 & 8.953 & 99.642 \\
\hline Line $10 \mathrm{~s}$ & 5.57 & 0.088 & 0.005 & & 09 & 0 & 0.481 & 57.509 & 0.024 & 9.047 & 99.767 \\
\hline S19-PI7-1 & 4.563 & 0.106 & 0.014 & 89 & 17 & 0 & 0.338 & 54.409 & 0.028 & 11.11 & 99.874 \\
\hline S19-P17-2 & 4.528 & 0.085 & 0.01 & 0.399 & 28.867 & 0.0 & 0.456 & 53.928 & 0.004 & 11.034 & 99.33 \\
\hline S19-PI8 & 4.84 & 0.066 & 0.005 & 0.499 & 28.136 & 0 & 0.442 & 54.442 & 0.033 & 10.503 & 98.966 \\
\hline S19-P19 & 4.835 & 0.063 & 0 & 0.507 & 28.14 & 0 & 0.426 & 55.528 & 0.033 & 10.471 & 100.003 \\
\hline S19-PI9-1 & 3.051 & 0.058 & 0 & 11.039 & 19.409 & 0.022 & 0.179 & 62.316 & 0.004 & 0.719 & 96.797 \\
\hline S19-P19-2 & 4.87 & 0.105 & 0.0 & 0.286 & 27.871 & 0 & 0.848 & 54.436 & 0.153 & 10.448 & 99.02 \\
\hline S19-PI10 & 4.397 & 0.095 & 0.002 & 0.475 & 28.279 & 0.027 & 0.647 & 53.668 & 0.115 & 11.056 & 98.761 \\
\hline S19-PI11 & 4.333 & 0.123 & 0.002 & 0.373 & 29.347 & 0.004 & 0.386 & 53.822 & 0.031 & 11.688 & 100.109 \\
\hline S19-PI12 & 4.906 & 0.074 & 0 & 0.378 & 28.032 & 0 & 0.415 & 55.403 & 0.021 & 10.729 & 99.958 \\
\hline S19-PI13 & 4.744 & 0.119 & 0 & 0.444 & 57 & 0 & 0.454 & 54.884 & 0.025 & 10.626 & 99.853 \\
\hline S19-Pl14 & 4.6 & 0.073 & 0.027 & 0.454 & 28.663 & 0 & 0.409 & 53.992 & 0.026 & 10.971 & 99.215 \\
\hline S19-PI14-2 & 3.369 & 0.086 & 0.003 & 0.261 & 30.915 & 0 & 0.378 & 51.424 & 0.039 & 13.292 & 99.767 \\
\hline S19 & 4.603 & 0.075 & 0 & & & 0.0 & 0.582 & 54.946 & 0.069 & 10.943 & 99.793 \\
\hline S19-P115core & 1.72 & 0.055 & 0.022 & 0.093 & 33.813 & 0 & 0.436 & 46.7 & 0 & 17.106 & 99.945 \\
\hline
\end{tabular}


Table 2. EPMA results of pyroxene in the gabbroic rocks.

\begin{tabular}{|c|c|c|c|c|c|c|c|c|c|c|}
\hline Sample & $\mathrm{TiO}_{2}$ & MnO & $\mathrm{K}_{2} \mathrm{O}$ & $\mathrm{Al}_{2} \mathrm{O}_{3}$ & $\mathrm{Cr}_{2} \mathrm{O}_{3}$ & $\mathrm{FeO}$ & $\mathrm{SiO}_{2}$ & MgO & $\mathrm{CaO}$ & Total \\
\hline S19-Px1 & 0.49 & 0.658 & 0 & 0.672 & 0 & 22.288 & 52.142 & 22.355 & 1.968 & 100.601 \\
\hline S19-Px1-inclu & 48.438 & 0.422 & 0.008 & 0.091 & 0.019 & 50.324 & 0.054 & 0.899 & 0.041 & 100.338 \\
\hline S19-Px2 & 0.908 & 0.314 & 0 & 1.847 & 0.024 & 11.155 & 51.528 & 14.425 & 19.348 & 99.777 \\
\hline S19-Px3 & 0.904 & 0.312 & 0 & 1.89 & 0.003 & 11.15 & 51.334 & 15.163 & 19.6 & 100.619 \\
\hline S19-Px3 & 0.903 & 0.306 & 0.006 & 1.902 & 0 & 11.225 & 50.924 & 15.076 & 19.633 & 100.238 \\
\hline S19-Px4 & 1.009 & 0.371 & 0.007 & 2.235 & 0.007 & 11.522 & 50.062 & 14.564 & 19.534 & 99.569 \\
\hline S19-Px4-2 & 0.907 & 0.323 & 0.002 & 2.01 & 0 & 11.39 & 50.726 & 14.83 & 19.507 & 99.997 \\
\hline S19-Px4-3 & 0.945 & 0.356 & 0.004 & 1.942 & 0.031 & 10.992 & 50.837 & 14.683 & 19.546 & 99.584 \\
\hline S19-Px6 & 0.773 & 0.243 & 0 & 2.948 & 0.012 & 9.694 & 50.322 & 14.77 & 20.787 & 99.781 \\
\hline S19-Px5 & 0.811 & 0.388 & 0.005 & 1.984 & 0.011 & 11.892 & 50.75 & 14.496 & 19.383 & 99.972 \\
\hline $\begin{array}{l}\text { S19-Px5- } \\
\text { inclu1 }\end{array}$ & 0.039 & 0.205 & 0.179 & 4.762 & 0.016 & 25.013 & 44.744 & 6.495 & 1.816 & 83.445 \\
\hline $\begin{array}{l}\text { S19-Px5- } \\
\text { inclu4 }\end{array}$ & 10.334 & 0.244 & 0.147 & 1.396 & 0.275 & 75.186 & 5.575 & 2.17 & 0.356 & 95.776 \\
\hline S19-Px8 & 0.884 & 0.341 & 0 & 1.72 & 0.02 & 11.737 & 51.504 & 14.955 & 19.462 & 100.919 \\
\hline $\begin{array}{l}\text { S19-Px8- } \\
\text { inclu3 }\end{array}$ & 10.403 & 0.493 & 0.012 & 0.436 & 0 & 18.537 & 42.162 & 12.062 & 16.352 & 100.54 \\
\hline S19-Px9 & 0.882 & 0.357 & 0.003 & 1.797 & 0 & 11.388 & 50.831 & 14.692 & 19.456 & 99.72 \\
\hline S19-Px10 & 0.899 & 0.337 & 0.002 & 1.744 & 0.037 & 11.447 & 50.972 & 14.574 & 19.608 & 99.876 \\
\hline S19-Px10 & 0.975 & 0.325 & 0 & 2.229 & 0.022 & 10.634 & 50.322 & 14.85 & 20.009 & 99.619 \\
\hline $\begin{array}{l}\text { S19-Px10- } \\
\text { inclu }\end{array}$ & 0.063 & 0.565 & 0 & 0.002 & 0.001 & 32.279 & 35.798 & 30.861 & 0.178 & 99.776 \\
\hline S19-Px11 & 0.429 & 0.625 & 0 & 0.761 & 0 & 22.258 & 52.399 & 22.447 & 2.03 & 100.977 \\
\hline S19-Px12 & 0.411 & 0.374 & 0 & 0.88 & 0 & 10.43 & 51.977 & 14.166 & 21.58 & 99.956 \\
\hline S19-Px13 & 0.994 & 0.364 & 0.006 & 2.363 & 0 & 11.167 & 50.008 & 14.432 & 19.772 & 99.387 \\
\hline S19-Px14 & 0.947 & 0.236 & 0 & 2.282 & 0.017 & 10.117 & 50.227 & 14.59 & 20.787 & 99.48 \\
\hline S19-Px15 & 1.24 & 0.246 & 0 & 3.002 & 0 & 10.384 & 49.919 & 14.659 & 20.163 & 99.897 \\
\hline S19-Px16 & 0.852 & 0.385 & 0.005 & 1.796 & 0.01 & 11.87 & 51.424 & 14.542 & 19.476 & 100.628 \\
\hline
\end{tabular}

Table 3. Plagioclase formula unit calculated on the basis of 8 oxygen atoms

\begin{tabular}{|c|c|c|c|c|c|c|c|c|}
\hline Sample & $\mathbf{S i}$ & $\mathbf{T i}$ & Al & $\mathbf{F e}($ ii) & $\mathbf{C a}$ & $\mathbf{N a}$ & $\mathbf{K}$ & TOTAL \\
\hline S19-PI1 & 9.53 & 0.01 & 6.48 & 0.05 & 2.39 & 1.43 & 0.06 & 19.96 \\
\hline S19-PI2 & 9.58 & 0.02 & 6.44 & 0.06 & 2.34 & 1.43 & 0.06 & 19.93 \\
\hline S19-PI2 & 9.61 & 0.02 & 6.38 & 0.07 & 2.37 & 1.39 & 0.09 & 19.92 \\
\hline S19-PI2-1 & 0.00 & 10.35 & 0.01 & 11.25 & 0.00 & 0.02 & 0.00 & 21.65 \\
\hline S19-PI2-2 & 0.01 & 10.39 & 0.01 & 11.18 & 0.01 & 0.00 & 0.00 & 21.60 \\
\hline
\end{tabular}




\begin{tabular}{|c|c|c|c|c|c|c|c|c|}
\hline Line 1 S19-P13 & 10.10 & 0.01 & 5.92 & 0.08 & 1.82 & 1.87 & 0.10 & 19.91 \\
\hline Line 2 S19-PI3 & 9.84 & 0.01 & 6.16 & 0.06 & 2.11 & 1.64 & 0.11 & 19.94 \\
\hline Line 3 S19-PI3 & 8.66 & 0.01 & 7.35 & 0.07 & 3.20 & 0.68 & 0.02 & 20.00 \\
\hline Line 4 S19-PI3 & 8.70 & 0.01 & 7.29 & 0.08 & 3.20 & 0.71 & 0.03 & 20.01 \\
\hline Line 5 S19-PI3 & 8.62 & 0.01 & 7.38 & 0.07 & 3.25 & 0.67 & 0.02 & 20.02 \\
\hline Line 6 S19-Pl3 & 8.70 & 0.01 & 7.30 & 0.07 & 3.18 & 0.71 & 0.03 & 20.01 \\
\hline Line 7 S19-PI3 & 8.61 & 0.01 & 7.38 & 0.08 & 3.29 & 0.63 & 0.02 & 20.02 \\
\hline Line 8 S19-PI3 & 8.72 & 0.01 & 7.27 & 0.07 & 3.18 & 0.72 & 0.03 & 20.01 \\
\hline Line 9 S19-PI3 & 10.59 & 0.01 & 5.44 & 0.05 & 1.39 & 2.23 & 0.15 & 19.87 \\
\hline Line 10 S19-PI3 & 10.04 & 0.01 & 5.99 & 0.06 & 1.89 & 1.87 & 0.09 & 19.94 \\
\hline Line 10 S19-PI4 & 10.05 & 0.01 & 5.96 & 0.07 & 1.89 & 1.86 & 0.09 & 19.93 \\
\hline S19-PI4-1 & 9.84 & 0.01 & 6.16 & 0.05 & 2.18 & 1.57 & 0.09 & 19.91 \\
\hline S19-PI4-2 & 8.74 & 0.01 & 7.22 & 0.07 & 3.24 & 0.69 & 0.03 & 20.00 \\
\hline Line 1 S19-PI5 & 10.38 & 0.00 & 5.63 & 0.07 & 1.62 & 2.06 & 0.11 & 19.88 \\
\hline Line 2 S19-P15 & 9.91 & 0.01 & 6.09 & 0.06 & 2.12 & 1.56 & 0.12 & 19.87 \\
\hline Line 3 S19-PI5 & 8.97 & 0.01 & 6.97 & 0.08 & 3.05 & 0.85 & 0.04 & 19.97 \\
\hline Line 4 S19-PI5 & 8.78 & 0.01 & 7.18 & 0.07 & 3.23 & 0.65 & 0.02 & 19.95 \\
\hline Line 5 S19-PI5 & 9.98 & 0.01 & 6.00 & 0.06 & 2.10 & 1.59 & 0.13 & 19.88 \\
\hline Line 6 S19-PI5 & 9.82 & 0.01 & 6.14 & 0.08 & 2.19 & 1.60 & 0.12 & 19.96 \\
\hline Line 7 S19-Pl5 & 9.78 & 0.01 & 6.22 & 0.06 & 2.26 & 1.43 & 0.11 & 19.88 \\
\hline Line 8 S19-P15 & 8.99 & 0.01 & 6.96 & 0.07 & 3.05 & 0.80 & 0.04 & 19.93 \\
\hline Line 9 S19-Pl5 & 10.01 & 0.01 & 5.98 & 0.06 & 2.03 & 1.72 & 0.12 & 19.92 \\
\hline Line 10 S19-PI5 & 10.34 & 0.01 & 5.64 & 0.08 & 1.73 & 1.96 & 0.10 & 19.86 \\
\hline Line 10 S19-P16 & 10.34 & 0.01 & 5.64 & 0.07 & 1.74 & 1.94 & 0.10 & 19.85 \\
\hline S19-Pl6(in)in Px6 & 10.49 & 0.00 & 5.52 & 0.05 & 1.58 & 2.06 & 0.15 & 19.85 \\
\hline S19-P17 & 8.93 & 0.01 & 7.03 & 0.09 & 3.06 & 0.82 & 0.03 & 19.97 \\
\hline S19-PI7-1 & 9.84 & 0.01 & 6.16 & 0.05 & 2.15 & 1.60 & 0.09 & 19.91 \\
\hline S19-P17-2 & 9.81 & 0.01 & 6.19 & 0.07 & 2.15 & 1.60 & 0.09 & 19.93 \\
\hline S19-PI8 & 9.94 & 0.01 & 6.05 & 0.07 & 2.05 & 1.71 & 0.12 & 19.94 \\
\hline S19-PI9 & 10.01 & 0.01 & 5.98 & 0.06 & 2.02 & 1.69 & 0.12 & 19.89 \\
\hline S19-PI9-1 & 11.73 & 0.01 & 4.31 & 0.03 & 0.15 & 1.11 & 2.65 & 19.99 \\
\hline S19-P19-2 & 9.95 & 0.01 & 6.00 & 0.13 & 2.05 & 1.73 & 0.07 & 19.93 \\
\hline S19-Px13 & 11.72 & 0.18 & 0.65 & 2.19 & 4.97 & 0.13 & 0.00 & 19.84 \\
\hline S19-PI10 & 9.85 & 0.01 & 6.11 & 0.10 & 2.17 & 1.56 & 0.11 & 19.92 \\
\hline S19-PI11 & 9.73 & 0.02 & 6.25 & 0.06 & 2.26 & 1.52 & 0.09 & 19.93 \\
\hline S19-PI12 & 10.00 & 0.01 & 5.96 & 0.06 & 2.07 & 1.72 & 0.09 & 19.91 \\
\hline S19-PI13 & 9.92 & 0.02 & 6.08 & 0.07 & 2.06 & 1.66 & 0.10 & 19.91 \\
\hline S19-PI14 & 9.84 & 0.01 & 6.16 & 0.06 & 2.14 & 1.63 & 0.11 & 19.94 \\
\hline S19-PI14-2 & 9.37 & 0.01 & 6.64 & 0.06 & 2.59 & 1.19 & 0.06 & 19.92 \\
\hline S19-PI15rim & 9.95 & 0.01 & 6.01 & 0.09 & 2.12 & 1.62 & 0.10 & 19.89 \\
\hline S19-PI15core & 8.60 & 0.01 & 7.34 & 0.07 & 3.38 & 0.61 & 0.02 & 20.03 \\
\hline
\end{tabular}


Table 4. formula unit calculated on the basis of Pyroxen 6 oxygen atoms

\begin{tabular}{|c|c|c|c|c|c|c|c|c|c|c|c|c|c|}
\hline Sample & Si & Al2 & Al & Fe(iii) & $\mathbf{C r}$ & $\mathbf{T i}$ & $\mathbf{F e}(\mathrm{ii})$ & Mn & Mg & $\mathbf{C a}$ & $\mathbf{N a}$ & $\mathbf{K}$ & TOTAL \\
\hline S19-Px1 & 1.945 & 0.030 & 0.000 & 0.083 & 0.000 & 0.014 & 0.608 & 0.021 & 1.243 & 0.079 & 0.002 & 0.000 & 4.023 \\
\hline S19-Px1-inclu & 1.945 & 0.030 & 0.000 & 0.083 & 0.000 & 0.014 & 0.608 & 0.021 & 1.243 & 0.079 & 0.002 & 0.000 & 4.023 \\
\hline S19-Px2 & 1.945 & 0.055 & 0.027 & 0.019 & 0.001 & 0.026 & 0.331 & 0.010 & 0.808 & 0.778 & 0.017 & 0.000 & 4.015 \\
\hline S19-Px3 & 1.915 & 0.083 & 0.000 & 0.081 & 0.000 & 0.025 & 0.264 & 0.010 & 0.843 & 0.783 & 0.019 & 0.000 & 4.025 \\
\hline S19-Px3 & 1.915 & 0.084 & 0.000 & 0.096 & 0.000 & 0.025 & 0.253 & 0.010 & 0.843 & 0.789 & 0.019 & 0.000 & 4.035 \\
\hline S19-Px4 & 1.896 & 0.100 & 0.000 & 0.105 & 0.000 & 0.029 & 0.257 & 0.012 & 0.822 & 0.792 & 0.019 & 0.000 & 4.032 \\
\hline S19-Px4-2 & 1.896 & 0.089 & 0.000 & 0.096 & 0.000 & 0.026 & 0.259 & 0.010 & 0.832 & 0.786 & 0.022 & 0.000 & 4.017 \\
\hline S19-Px4-3 & 1.917 & 0.083 & 0.003 & 0.066 & 0.001 & 0.027 & 0.279 & 0.011 & 0.825 & 0.790 & 0.018 & 0.000 & 4.020 \\
\hline S19-Px6 & 1.917 & 0.083 & 0.047 & 0.097 & 0.000 & 0.022 & 0.205 & 0.008 & 0.826 & 0.836 & 0.017 & 0.000 & 4.058 \\
\hline S19-Px5 & 1.913 & 0.087 & 0.002 & 0.085 & 0.000 & 0.023 & 0.287 & 0.012 & 0.815 & 0.783 & 0.018 & 0.000 & 4.026 \\
\hline S19-Px5-inclu1 & 2.050 & -0.050 & 0.307 & 0.000 & 0.001 & 0.001 & 1.000 & 0.008 & 0.444 & 0.089 & 0.016 & 0.010 & 3.875 \\
\hline S19-Px5-inclu4 & 0.347 & 0.102 & 0.000 & 2.629 & 0.014 & 0.484 & 0.427 & 0.013 & 0.201 & 0.024 & 0.011 & 0.012 & 4.265 \\
\hline S19-Px8 & 1.920 & 0.076 & 0.000 & 0.082 & 0.001 & 0.025 & 0.281 & 0.011 & 0.831 & 0.777 & 0.021 & 0.000 & 4.025 \\
\hline S19-Px8-inclu3 & 1.662 & 0.020 & 0.000 & 0.070 & 0.000 & 0.308 & 0.537 & 0.016 & 0.709 & 0.690 & 0.006 & 0.001 & 4.020 \\
\hline S19-Px9 & 1.662 & 0.080 & 0.000 & 0.086 & 0.000 & 0.025 & 0.270 & 0.011 & 0.826 & 0.786 & 0.023 & 0.000 & 3.770 \\
\hline S19-Px10 & 1.920 & 0.077 & 0.000 & 0.073 & 0.001 & 0.025 & 0.286 & 0.011 & 0.819 & 0.791 & 0.019 & 0.000 & 4.022 \\
\hline S19-Px10 & 1.898 & 0.099 & 0.000 & 0.100 & 0.001 & 0.028 & 0.233 & 0.010 & 0.835 & 0.809 & 0.019 & 0.000 & 4.031 \\
\hline S19-Px11 & 1.946 & 0.033 & 0.000 & 0.080 & 0.000 & 0.012 & 0.607 & 0.020 & 1.243 & 0.081 & 0.002 & 0.000 & 4.022 \\
\hline S19-Px12 & 1.955 & 0.039 & 0.000 & 0.057 & 0.000 & 0.012 & 0.269 & 0.012 & 0.794 & 0.869 & 0.010 & 0.000 & 4.018 \\
\hline S19-Px13 & 1.895 & 0.105 & 0.001 & 0.101 & 0.000 & 0.028 & 0.250 & 0.012 & 0.815 & 0.803 & 0.021 & 0.000 & 4.031 \\
\hline S19-Px14 & 1.897 & 0.102 & 0.000 & 0.105 & 0.001 & 0.027 & 0.211 & 0.008 & 0.821 & 0.841 & 0.020 & 0.000 & 4.033 \\
\hline S19-Px15 & 1.876 & 0.124 & 0.009 & 0.097 & 0.000 & 0.035 & 0.227 & 0.008 & 0.821 & 0.812 & 0.021 & 0.000 & 4.030 \\
\hline S19-Px16 & 1.924 & 0.076 & 0.003 & 0.066 & 0.000 & 0.024 & 0.303 & 0.012 & 0.811 & 0.781 & 0.019 & 0.000 & 4.020 \\
\hline
\end{tabular}

Table 5. EPMA results of olivine, K-feldspar and opaque minerals in the gabbroic rocks.

\begin{tabular}{llllllllllll}
\hline \multicolumn{1}{c}{ Sample } & $\mathbf{N a 2 O}$ & $\mathbf{T i O 2}$ & $\mathbf{M n O}$ & $\mathbf{K 2 O}$ & $\mathbf{A l 2 O 3}$ & $\mathbf{C r 2 O 3}$ & $\mathbf{F e O}$ & $\mathbf{S i O 2}$ & $\mathbf{M g O}$ & $\mathbf{C a O}$ & Total \\
S19-Px10-inclu & 0.029 & 0.063 & 0.565 & 0 & 0.002 & 0.001 & 32.279 & 35.798 & 30.861 & 0.178 & 99.776 \\
S19-Px10-inclu & 0.038 & 0.06 & 0.582 & 0.006 & 0.018 & 0 & 32.356 & 36.339 & 30.887 & 0.169 & 100.455 \\
Opaque & & & & & & & & & & & \\
mineral & $\mathrm{Na} 2 \mathrm{O}$ & $\mathrm{TiO} 2$ & $\mathrm{MnO}$ & $\mathrm{K} 2 \mathrm{O}$ & $\mathrm{Al} 2 \mathrm{O} 3$ & $\mathrm{Cr} 2 \mathrm{O} 3$ & $\mathrm{FeO}$ & $\mathrm{SiO} 2$ & $\mathrm{MgO}$ & $\mathrm{CaO}$ & $\mathrm{Total}$ \\
$\mathbf{S 1 9 - P 1 2 - 1}$ & 0.044 & 51.085 & 0.449 & 0.004 & 0.044 & 0 & 49.925 & 0.015 & 0.688 & 0.014 & 102.268 \\
S19-P12-2 & 0 & 51.216 & 0.458 & 0.011 & 0.037 & 0.017 & 49.546 & 0.025 & 0.704 & 0.024 & 102.038 \\
S19-Px1-inclu & 0.042 & 48.438 & 0.422 & 0.008 & 0.091 & 0.019 & 50.324 & 0.054 & 0.899 & 0.041 & 100.338 \\
K-feldspar & $\mathrm{Na} 2 \mathrm{O}$ & $\mathrm{TiO} 2$ & $\mathrm{MnO}$ & $\mathrm{K} 2 \mathrm{O}$ & $\mathrm{Al} 2 \mathrm{O} 3$ & $\mathrm{Cr} 2 \mathrm{O} 3$ & $\mathrm{FeO}$ & $\mathrm{SiO} 2$ & $\mathrm{MgO}$ & $\mathrm{CaO}$ & $\mathrm{Total}$ \\
S19-P19-1 & 3.051 & 0.058 & 0 & 11.039 & 19.409 & 0.022 & 0.179 & 62.316 & 0.004 & 0.719 & 96.797 \\
\hline
\end{tabular}




\section{Plagioclase}

The formula unit of plagioclase is calculated based on 8 oxygen atoms (Table 1). The analysed plagioclase crystals plot in the andesine, labradorite, and bytonite fields in Or-Ab-An diagram (Figure. 4) of Deer et al. (1991). The compositional range for plagioclase is $\mathrm{An}_{14.22} \mathrm{Ab}_{80.92} \mathrm{Or}_{4.86}$ to $\mathrm{An}_{84.14} \mathrm{Ab}_{15.31} \mathrm{Or}_{0.55}$. K-feldspar has a composition of $\mathrm{Or}_{73} \mathrm{Ab}_{26} \mathrm{An}_{1}$. Plagioclase crystals show chemical zoning, which is due to $\mathrm{Al} / \mathrm{Si}$ ratio variation in the magma during crystallization. This shows sluggish equilibrium rate compared to crystallization rate (Shelly, 1993). Analyzed samples show oscillatory (Figure. 5 a and b) and normal (Figure. 5c) chemical zoning. Anorthite content decreases from the core to the rim, indicating a normal zoning. It shows almost rhythmic decrease and increase in grains with oscillatory zoning. The reason for zoning is either crystallization from a magma with changing temperature, vapour pressure and chemistry (as a result of magma mixing and/or magma chemical changes during crystallization, Humphreys et al., 2006). Pressure decreasing owing to rapid magma ascend and changes in oxygen fugacity also can cause zoning in plagioclase crystallizing from a magma (Blundy et al., 2006). Magma injection into the magma chambers as intervals causes changes in the oxygen fugacity and therefore formation of chemical zoning in plagioclase minerals. Increase in growth rate at the boundary between crystal and melt also can cause plagioclase chemical zoning (Ginibre et al., 2002).

\section{Pyroxene}

The formula unit of pyroxene is calculated based on six oxygen atoms and 4 cations. Criteria by of Morimoto et al. (1988) was used for classification of the analysed pyroxenes (Figure. 5a). The data plot in the Fe-Mg-Ca pyroxenes in the Q-J diagram of Figure. 5a. They are augite, hypersthene and ferrohypersthene (Figure. 5b) according to classification of Poldervaart and Hess (1951). The compositional variation of pyroxene phenocrysts in the studied rocks is $\mathrm{En}_{0.44} \mathrm{Fs}_{5.07} \mathrm{Wo}_{94.50}$ and $\mathrm{En}_{61.14} \mathrm{Fs}_{34.98} \mathrm{Wo}_{3.86}$.

\section{Olivine}

The composition of analysed olivines are mainly hyalosiderite (Figure.6) according to Wager and Deer (1939).



figure 4. Plagioclase composition in Ab-An-Or ternary diagram. 

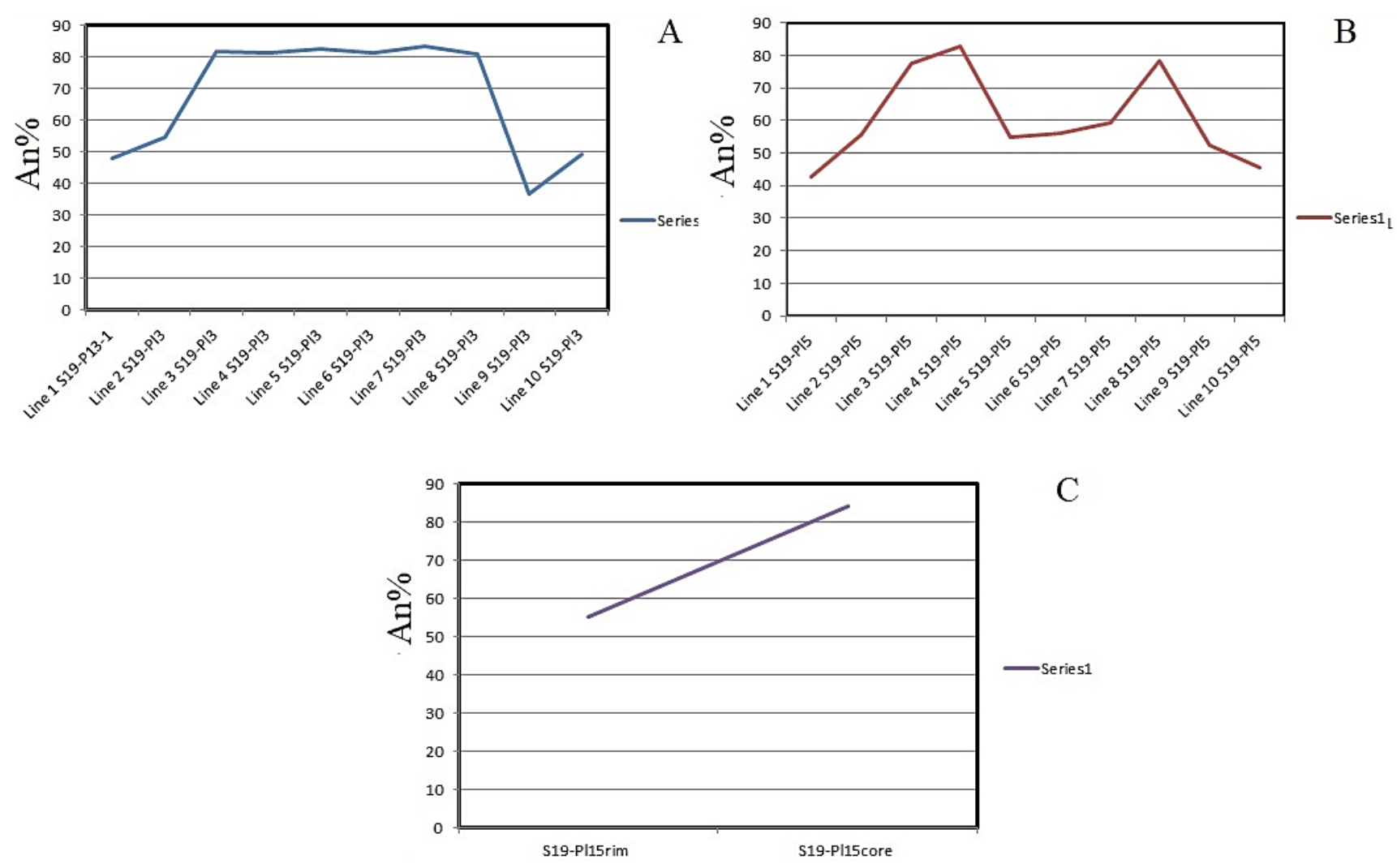

figure 5. Zoning profile across three plagioclase crystals.
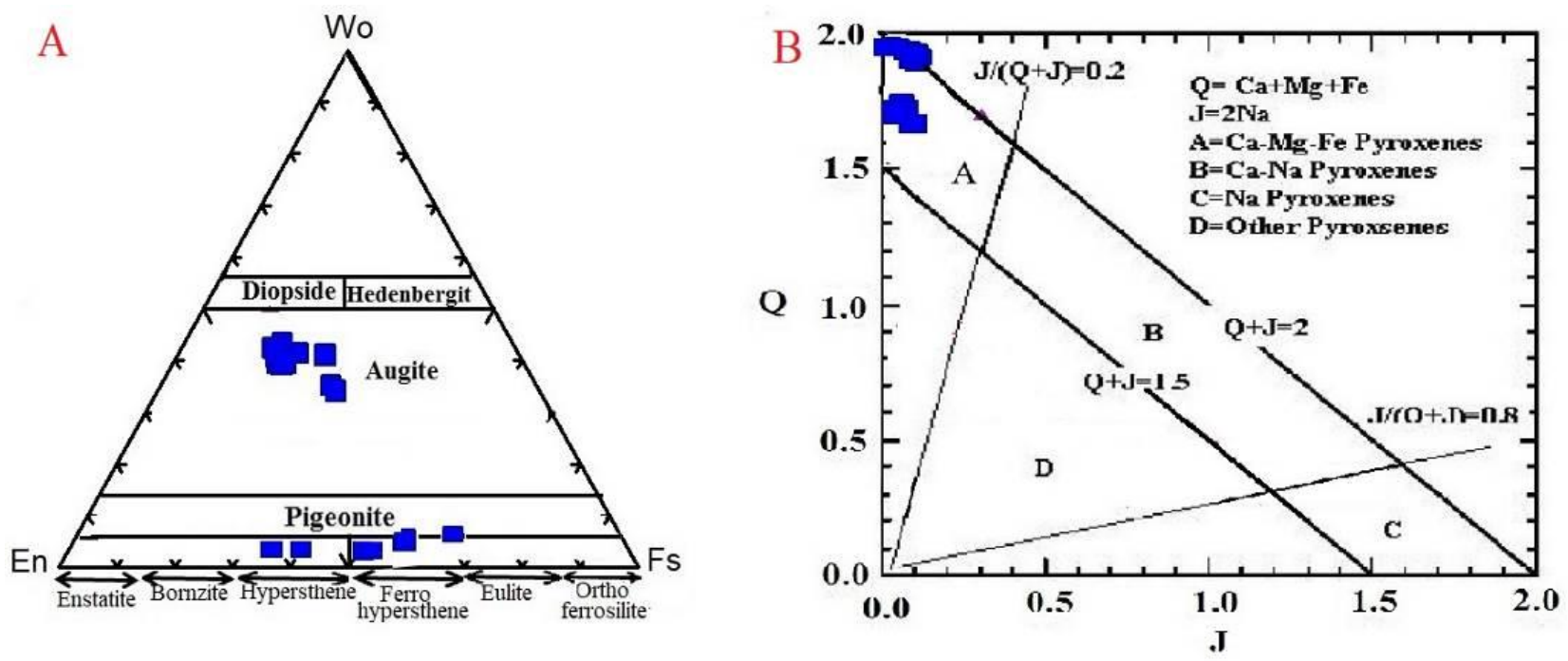

figure 6. A: Pyroxene classification in Q-J diagram. B: Pyroxene classification in the Poldervaart and Hess (1951) diagram. 


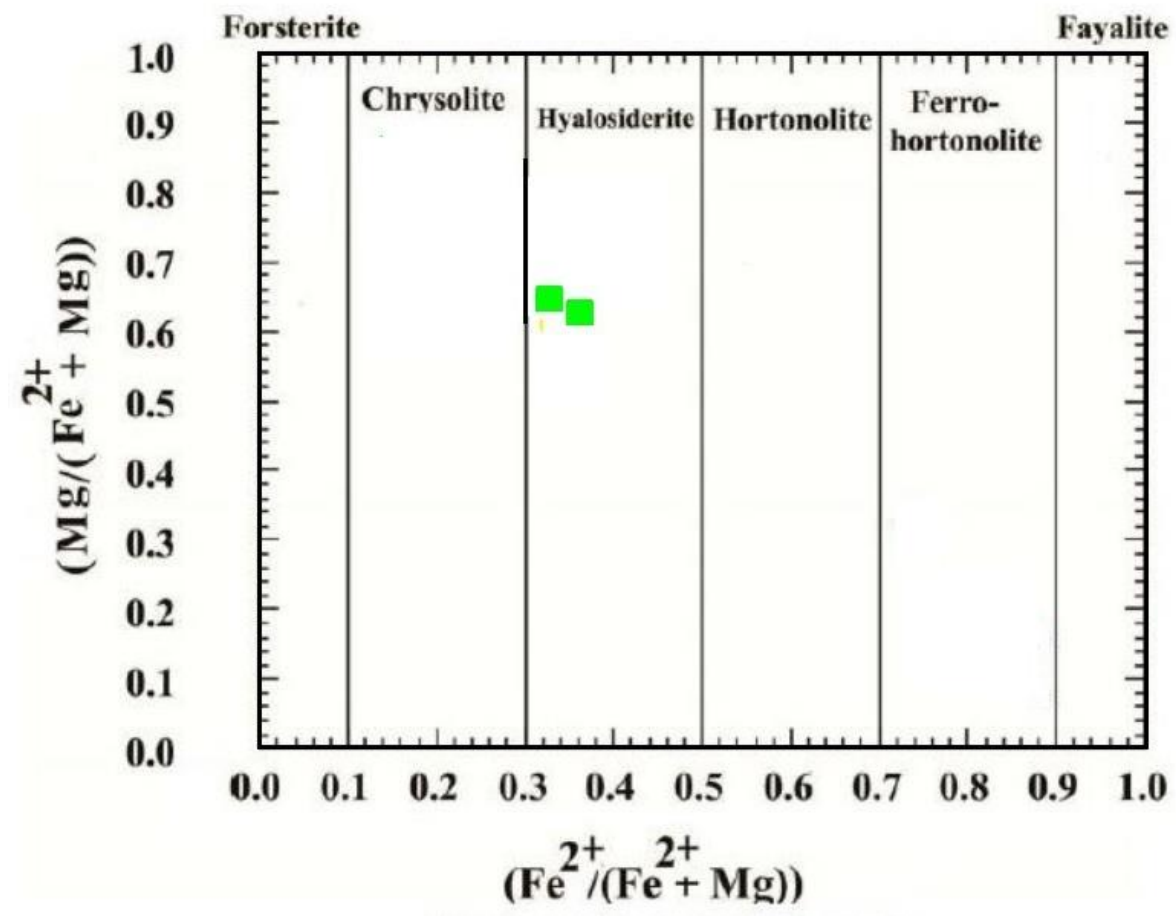

figure 7. All analysed olivines are hyalosiderite.

\section{PRESSURE AND TEMPERATURE (P-T) ESTIMATIONS}

Pyroxene chemistry is a powerful tool or pressure and temperature estimation of crystallization. Different geothermobarometers were applied to find the P-T condition of crystallization of the studied gabbro samples. Calibration by Soesso (1997) gives temperatures of $1100{ }^{\circ} \mathrm{C}$ to $1150{ }^{\circ} \mathrm{C}$ and pressures of 1 to 3 kbar for pyroxene crystallization (Figure. $8 \mathrm{a}$ and b). Two pyroxene thermometry of Lindsley et al. (1983) gives temperatures of $950^{\circ} \mathrm{C}$ to $1130^{\circ} \mathrm{C}$ (Figure.8c), which is in good agreement with results from thermometer of Soesso (1997). Normative percentage of Or-Ab-An diagram indicates temperatures of $650^{\circ} \mathrm{C}$ to $750^{\circ} \mathrm{C}$ for feldspar crystallization in the rocks.

Oxygen fugacity is an important controlling factor for liquidus temperature and melt/crystal composition (France et al., 2010) and magmatic processes (Kilinc et al., 1983; Kress and Carmichael, 1991; Ottonello et al., 2001; Botcharnikov et al., 2005; Moretti, 2005).

Using $\mathrm{Al}^{\mathrm{IV}}+\mathrm{Na}$ versus $\mathrm{Al}^{\mathrm{VI}}+2 \mathrm{Ti}+\mathrm{Cr}$, which is a function of $\mathrm{Fe}^{3+}$ content of pyroxene, oxygen fugacity during pyroxene crystallization can be estimated. A diagram is constructed (Schweitzer et al., 1979) based on $\mathrm{Al}$ in tetrahedral and octahedral crystallographic sites. $\mathrm{Fe}^{3+}$ can substitute for $\mathrm{Al}^{\mathrm{VI}}$, $\mathrm{Ti}$ and $\mathrm{Cr}$ in the octahedral site. Therefore $\mathrm{Fe}^{3+}$ content in pyroxene is a $\mathrm{Al}^{\mathrm{VI}}$ content. Data plotting above the $\mathrm{Fe}^{3+}=0$ line in the diagram (Figure. 9) are for pyroxenes crystallized at high $f_{\mathrm{O} 2}$. Papike and Cameron (1981) pointed out that samples plotting away from the line show higher $f_{\mathrm{O} 2}$. Our samples plot below the $\mathrm{Fe}^{3+}=0$, indication low $f_{\mathrm{O} 2}$ during crystallization (Figure.9). 

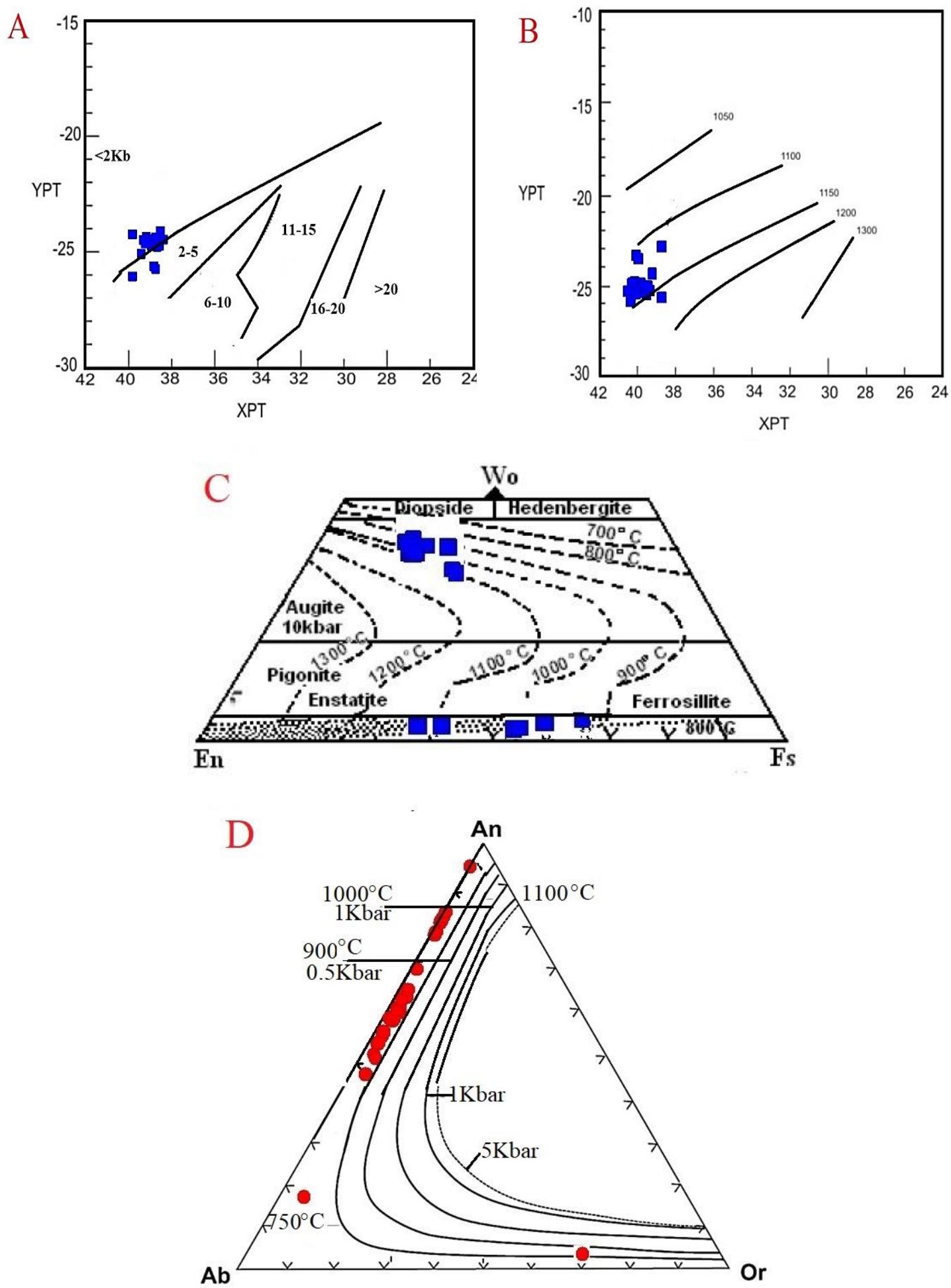

figure 8. A and B: Estimating pyroxene pressure crystallization using Soesso (1997) method. C: Temperature estimation using method of Lindsley et al. (1983). D: Or-Ab-An diagram for temperature estimation of feldspar crystallization (after Seck, 1971). 




figure 9. $f_{\mathrm{O} 2}$ condition for magma crystallization using diagram of Schweitzer et al. (1979).

\section{MAGMATIC NATURE AND TECTONIC SETTING}

Chemistry of clinopyroxene is used to find out the magmatic nature and tectonic setting for the studied gabbros (e.g Moazzen and Oberhänsli, 2008). Al and Ti content in clinopyroxene depends on silica content of magma and the ratio of these elements in tholeiitic, alkaline and calc-alkaline magmas (Kushiro,1960; Le Bas, 1962). The analysed pyroxenes plot on and above the saturation line of diagram in Fig. 10. This shows that the tetrahedral site is occupied by $\mathrm{Si}$ and $\mathrm{Al}^{\mathrm{IV}}$ (Schweitzer et al., 1979). Ti content in clinopyroxene is related to the alkalinity of parental magma. Clinopyroxenes in alkaline basalts contain higher amount of $\mathrm{Ti}$, compared to sub-alkaline basalts (Gill, 2010). Our samples plot in the sub-alkaline field of $\mathrm{SiO}_{2}$ versus $\mathrm{Al}_{2} \mathrm{O}_{3}$ and $\mathrm{Al}_{2} \mathrm{O}_{3}$ versus $\mathrm{TiO}_{2}$ diagrams of Le Bas (1962) in Fig. 11a and b. Clinopyroxene compositions in diagrams by Leterrier et al (1982) indicate calc-alkaline nature for the parental magma of the gabbroic rocks and a volcanic arc tectonic setting (Fig. 11c, d and e). Ti-Ca criteria of Leterrier et al. (1982), confirms an orogenic setting for the gabbros (Fig. 11e).

\section{CONCLUSIONS}

The gabbroic rocks of the Gysel area of Alborz Mountains in north Iran are gabbro porphyry, olivine gabbro, olivine dolerite and olivine monzo-gabrro. The main mineral consitient is plagioclase and pyroxene with subordinate amounts of altered olivine. Plagioclase composition is labradorite to bytonite with oscillatory and normal zoning, clinopyroxene is augite and orthopyroxene is hypersthene to ferrohypersthene. Olivine is hyalosiderite. P-T estimations indicate 650 to 750 for plagioclase crystallization and 950 to 1150 for pyroxene crystallization. Clinopyroxene composition reveals a calc-alkaline nature for the magma and volcanic arc tectonic setting. 


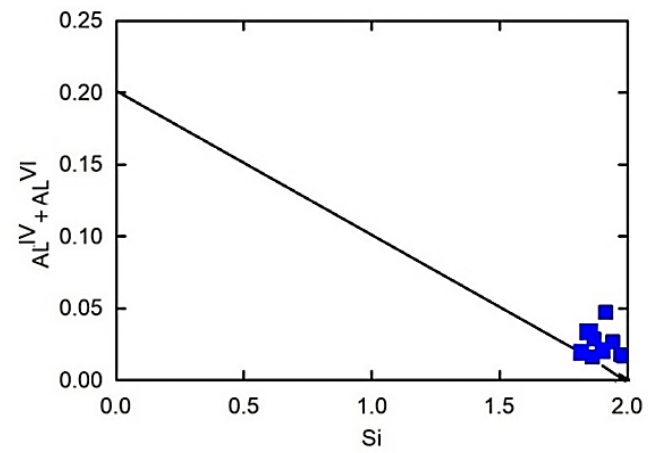

figure 10. $\mathrm{Al}^{\mathrm{IV}}+\mathrm{Al}^{\mathrm{VI}}$ versus Si diagram for the analysed clinopyroxenes.
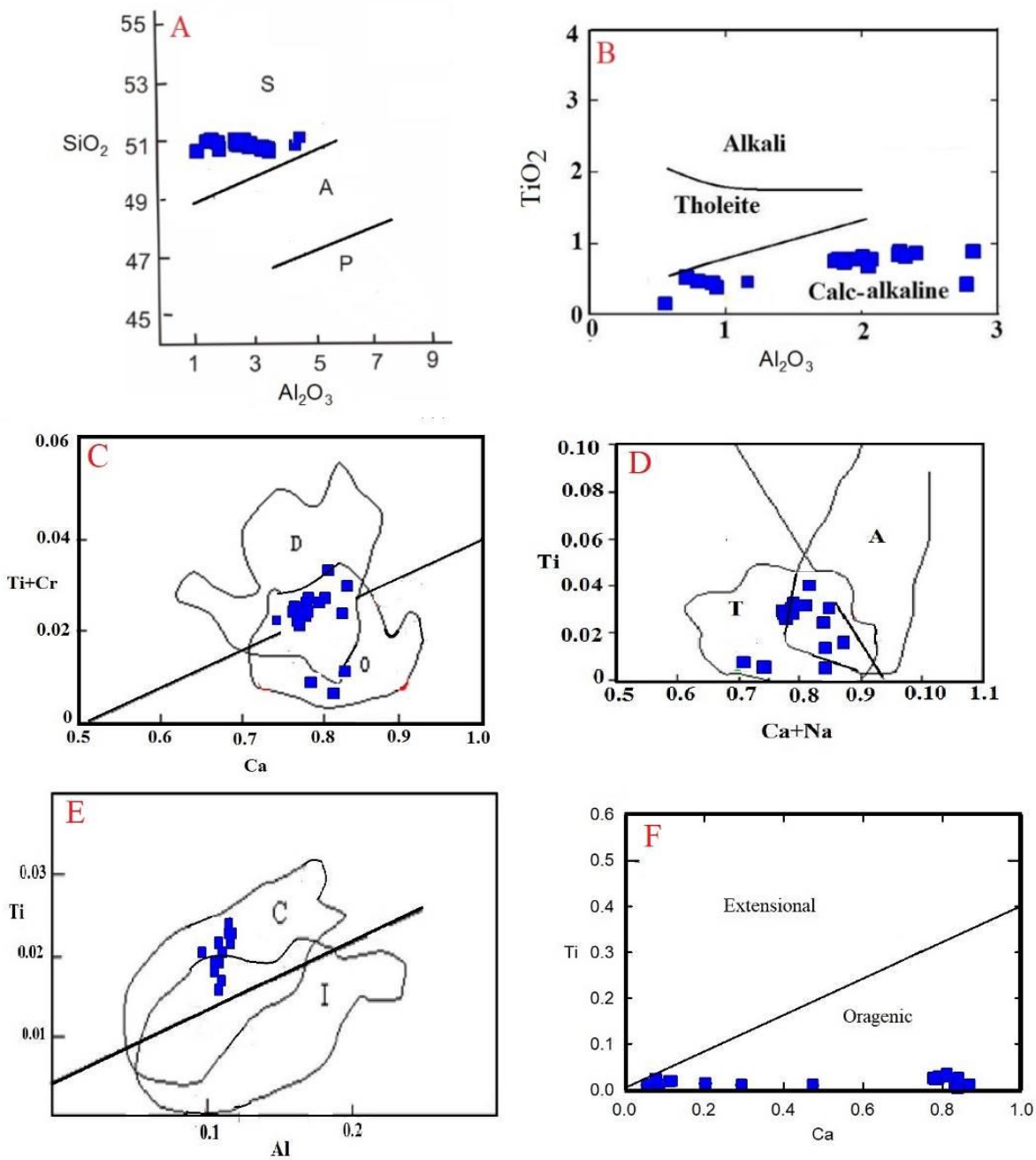

figure 11. Plotting of pyroxene analyses on $\mathrm{Al}_{2} \mathrm{O}_{3}$ versus $\mathrm{SiO}_{2}$ and $\mathrm{Al}_{2} \mathrm{O}_{3}$ versus $\mathrm{TiO}_{2}$ diagrams (Le $\mathrm{Bas}, 1962$ ) (A and B) for distinguishing the nature of the parental magma. C, D and E: diagrams from Leterrier et al. (1982) for magma type and tectonic setting ( $\mathrm{T}=$ Island arc tholeiitic basalt, $\mathrm{A}=$ Alkali basalt, $\mathrm{O}=$ volcanic arc basalt, $\mathrm{D}=$ MORB, I+ Island arc tholeiite, $\mathrm{C}=\mathrm{Calc}$-alkaline basalt. $\mathrm{F}$ : Ca versus Ti diagram showing tectonic setting after Leterrier et al. (1982). 


\section{ACKNOWLEDGEMENTS}

We thank Mr. Mahdi Asadian for his help during field studies. Mohssen Moazzen is acknowledged for scientific inputs. Dr. Rezaii helped with electron microprobe analyses in the Potsdam University.

\section{REFERENCIAS}

Agard P., Omrani J., Jolivet L., Whitechurch H., Vrielynck B., Spakman W., Monie P., Meyer B., (2011). Zagros orogeny: a subductiondominated process. Geological Magazine, 148, 692-725.

Wortel R.,

Annells R.N., Arthurton R.S., Bazely R.A., Davies R.G., (1975). Explanatory text of the Qazvin and Rasht quadrangle map 1:250000". Geological Survey of Iran, Reports E3-E4, 108.

Asiabanha A., (2001). Geology and petrogenesis of volcanic facies at the Uzbashichai area, west of Qazvin. PhD thesis, University of Tarbiyat Modares, 321.

Asiabanha A., Foden J., (2012). Post-collisional transition from an extensional volcano-sedimentary basin to a continental arc in the Alborz Ranges, N-Iran", Lithos, 148, 98-111.

Baharfirouzi, K., Shafei, A., Azhdari, A. and Karimi, H. R., (2002). Geological Map of Javaherdeh 1:100,000. Geological Survey of Iran, Tehran.

Berger J., Femenias O., Mercier J.C.C., Demaiffe D.,(2005). Ocean-floor hydrothermal metamorphism in the Limousin ophiolites (western French Massif Central): evidence of a rare preserved Variscan oceanic marker. Journal of Metamorphic Geology, 23 , 795- 812.

Blundy J., Cashman K., Humphreys M., (2006). Magma heating by decompression-driven crystallization beneath andesite volcanoes. Nature, 443, 76-80.

Botcharnikov, R.E., Koepke, J., Holtz, F., McCammon, C., Wilke, M., (2005). The effect ofwater activity on the oxidation and structural state of Fe in a ferro-basaltic melt. Geochim. Cosmochim. Acta 69, 5071-5085.

Cameron, M., Papike, JJ., (1981). Structural and chemical variations in pyroxenes. AmericanMineralogist 66: 1-50.

Deer, W.A., Howie, R.A., and Zussman, J., (1991). An introduction to rock forming minerals. Longman., 528p.

Dioh E., Béziat D., Grégoire M, Debat P., (2009). Origin of rare earth element variations in clinopyroxene from plutonic and associated volcanic rocks from the Foulde basin, Northern Kedougou inlier, Senegal, West Africa. European Journal of Mineralogy, 21, 1029-1043.

France, L., Ildefonse, B., Koepke, J. and Bech, F., (2010). A new method to estimate the oxidation state of basaltic series from microprobe analysis. Journal of Volcanology and Geochemistry 69 61-120.

Gile, H. A., Boni, M., Balssone, G., Allen, C.R.,Banks, D. \& Moore, F., (2006). Marble-hosted sulfide ores in the Angouran $\mathrm{Zn}-(\mathrm{Pb}-\mathrm{Ag})$ deposit, NW Iran: interaction of sedimentary brines with a metamorphic core complex. Mineralium Deposita 41, 1-16.

Gill, R., (2010). Igneous rocks and processes a practical guide. Department of Earth Sciences Royal Holloway University of London, $472 \mathrm{pp}$.

Ginibre C., Kronz A., Wörner G., (2002). High-resolution quantitative imaging of plagioclase composition using accumulated backscattered electron images: new constraints on oscillatory zoning. Contributions to Mineralogy and Petrology", 142, 436-448.

Ginibre C., Kronz A., Worner G., (2002). Minor and trace-element zoning in plagioclase: implicationsfor magma chamber processes at Parinacota volcano, northern Chile. Contributions to Mineralogy and Petrology, 143, 300-315.

Humphreys M.C.S., Blundy J.D., Sparks S.J., (2006). Magma evolution and opensystem processes at Shiveluch volcano: insights from phenocryst zoning. Journal of Petrology, 47 (12), 2303-2334.

Kilinc, A., Carmichael, I.S.E., Rivers, M.L., Sack, R.O., (1983). The ferric-ferrous ratio of natural silicate liquids equilibrated in air. Contrib. Mineral. Petrol. 83, 136-140.

Kress, V.C., Carmichael, I.S.E., (1991). The compressibility of silicate liquids containingFe2O3and the effect of composition, temperature, oxygen fugacity and pressure on their redox states. Contrib. Mineral. Petrol. 108, $82-92$.

Kushiro I., (1960). Si-Al relation in clinopyroxenes from igneous rocks. American Journal of Science, 258, 548-554.

Le Bas M. J., (1962). The role of aluminium in igneous clinopyroxenes with relation to their parentage, American Journal of Scince 260, 267-288.

Le Bas, M.J., (1962). The role of aluminum in igneous. clinopyroxenes with relation to their parentage. American Journal of Science., 260, 267-288. 
Leterrier J., Maury R.C., Thonon P., Girard D., Marchal M., (1982). Clinopyroxene composition as amethod of identification of the magmatic affinities of paleo-volcanic series", Earth an Planetary Science Letters, 59,139154.

Lindsley D. H., (1983). Pyroxene thermometry. Americam Mineralogist ,68, 477-493.

Moazzen, M., Oberhänsli, R., (2008). Whole rock and relict igneous clinopyroxene geochemistry of ophiolite-related amphibolites from NW Iran - Implications for protolith nature. Neues Jahrbuch für Mineralogie Abhandlungen,185, (1), 51-62(12)

Mohajjel,M., Fergusson, CL., (2014). Jurassic to Cenozoic tectonics of the Zagros Orogen in northwestern Iran. International Geology Review, 56 (3), 263-287.

Moretti, R., (2005). Polymerization, basicity, oxidation state and their role in ionic modelling of silicate melts. Geophysics, 48(4- 5), 583-608.

Morimoto, N. (1989) Nomenclature of pyroxenes. The Canadian Mineralogist 27(1): 143-156.

Nemati B., Asiabanha A., (2017). Volcano-plutonic relations in Lat-Bolukan district (North of Qazvin, Western Alborz): Petrogenetic analysis and geochemical modeling, Journal of Petrology, 8 (31), 167-180.

Ottonello, G., Moretti, R., Marini, L., Zuccolini, M.V., (2001). Oxidation state of iron insilicate glasses and melts: a thermochemical model. Chem. Geol. 174, 157-179.

Poldervaart, A.; Hess, H. H., (1951). Pyroxenes in the Crystallization of Basaltic Magma. The Journal of Geology, $59,(5), 472-489$.

Putrika K.D., (2008). Thermometers and Barometers for Volcanic Systems", Reviews in Mineralogy and Geochemistry, 69, 61-120.

Putirka K.D., Mikaelian H., Ryerson F., Shaw H., (2003). New clinopyroxene-liquid thermobarometers for mafic evolved and volatile bearing lava compositions, with applications to lavas from Tibet and Snake River Plain, Idaho. American Mineralogist, 88, 1542-1554.

Rahimi G., Kananian A., Asiabanha A., (2010). Tectonic setting and petrogenesis of post-Eocene

volcanic rocks of Abazar district (NE of Qazvin). Journal of Crystallography and Mineralogy, 18(2), 167-180 (in Persian).

Schweitzer E. L., Papike J. J., Bence A. E., (1979). Statistical analysis of clinopyroxenes from deep sea basalts. American Mineralogist, 64,501-13.

Schweitzer, E. L., Papike, J. J. and Bence, A. E., 1979. Statistical analysis of clinopyroxenes from deep sea basalts. American Mineralogist 64: 502-513.

Seck H. A., (1971), Koexistierende Alkalifeldspate and Plagioklase in system $\mathrm{NaAlSi}_{3} \mathrm{O}_{8}-\mathrm{KAlSi}_{3} \mathrm{O}_{8}$ $\mathrm{CaAl}_{2} \mathrm{Si}_{2} \mathrm{O}_{8}$ bei Temperaturen $650^{\circ} \mathrm{C}$ bis $900^{\circ} \mathrm{C}$. Neues Jahrbuch fur Mineralogie Abhandlungen, $115,315-$ 34.

Shelly, D.. (1993). Igneous and metamorphic rocks under microscope classification features, microstructures and mineral preferred orientations. Chapman \& Hall, London, 405 pp.

Soesoo A. A., (1997). multivariate statistical analysis of clinopyroxene composition: empirical coordinates for the crystallisation P-T estimations. Geological Society of Sweden (Geologiska Föreningen) 119,55-60.

Teimouri S., Ghasemi H., Asiabanha A., (2018). The role of crustal contamination and differentiation in the formation of the Eocene volcanic rocks in Jirande area (Northwest of Qazvin). Journal of Petrology, 9 (33), 71-90.

Valizadeh M.V., Abdollahi H.R., Sadeghian M., (2008). Geological investigations of main intrusions of the Central Iran. Geosiences Scientific Quarterly Journal, 17(67),182-197 (in Persian).

Verdel, C., Wernicke, B.P., Hassanzadeh, J., (2011). A Paleogene extensional arc flare-up in Iran,Tectonics, 30, Issue 3.

Wager, L. R., Deer, W. A., (1939). The petrology of the Skaergaard intrusion, Kangerdlugssuak, East Greenland. Meddel. om Grenland, 105, 1-352. 